\title{
Carcinosarcoma of Stomach Confined to the Mucosa
}

\author{
Hirohiko Kuroda,* Hiroaki Saito,* Yusuke Kono,* Yuki Murakami,* Yuji Shishido,* Tomoyuki Matsunaga,* Yoji \\ Fukumoto,* Tomohiro Osaki,* Keigo Ashida,* Kanae Nosaka, $\dagger$ Yoshihisa Umekita $\dagger$ and Yoshiyuki Fujiwara* \\ *Division of Surgical Oncology, Department of Surgery, School of Medicine, Tottori University Faculty of Medicine, Yonago 683-8504, \\ Japan, and †Division of Organ Pathology, Department of Pathology, School of Medicine, Tottori University Faculty of Medicine, Yona- \\ go 683-8504, Japan
}

\begin{abstract}
Carcinosarcoma is a malignant tumor composed of both epithelial and mesenchymal malignant tumor components. A 78-year-old man was transferred to our hospital because of hematemesis and tarry stool. An emergency gastrointestinal endoscopic examination revealed active bleeding from an ulcerative lesion on the posterior wall of the gastric body; endoscopic hemostasis was successfully performed. A gastrointestinal endoscopy performed two months later showed a polypoid lesion at the same place where the ulcer had been. The biopsy specimen was histologically diagnosed as well to moderately differentiated tubular adenocarcinoma. The patient underwent a laparoscopic distal gastrectomy with D2 lymph node dissection under a diagnosis of gastric adenocarcinoma. A $28 \times 15 \mathrm{~mm}$ polypoid tumor was resected from the gastric body, and was found on microscopic examination to consist of both carcinoma and sarcoma components, showing atypical spindle cells, which were positive for $\alpha$-smooth muscular actin, calponin, and h-caldesmon, but negative for CD34, CD117 (c-kit), desmin, and dog 1 . These findings led to a diagnosis of gastric carcinosarcoma. The tumor was confined to the mucous membrane. Lymph node metastasis was found in one node and contained only the carcinoma component. The postoperative course was uneventful. The patient lived without recurrence for 2 years. Carcinosarcoma of the stomach is a rare tumor with high malignant potential and poor prognosis. Careful follow up is required for early detection of any recurrence.
\end{abstract}

Key words carcinosarcoma; gastric cancer; stomach

Carcinosarcoma is a malignant tumor composed of both epithelial and mesenchymal malignant tumor compo-

Corresponding author: Hiroaki Saito, MD, PhD

sai10@med.tottori u.ac.jp

Received 2017 September 20

Accepted 2017 November 6

Abbreviations: CA, carbohydrate antigen; $\mathrm{CD}$, cluster of differentiation; CEA, carcinoembryonic antigen; CT, computed tomography; IHC, immunohistochemical; SMA, smooth muscular actin nents. ${ }^{1}$ It typically occurs in the uterus, esophagus, and breasts, but only about 70 cases have been reported in the stomach to date..$^{2-8}$ Most cases are diagnosed at advanced stages, which indicates that this is a rapidly progressing tumor. ${ }^{9} 10$ Here, we report a case of gastric carcinosarcoma confined to the mucosa of the gastric wall. To our knowledge, this is the first case in which gastric carcinosarcoma was confined to the gastric mucosa.

\section{PATIENT REPORT}

A 78-year-old man was transferred to our hospital because of hematemesis and tarry stool. Laboratory examinations revealed moderate anemia (hemoglobin: $9.2 \mathrm{~g} /$ dL). An emergent gastrointestinal endoscopic examination revealed active bleeding from an ulcerative lesion on the posterior wall of the gastric body; endoscopic hemostasis was successfully performed. A proton pump inhibitor (esomeprazole magnesium hydrate) was given to treat the gastric ulcer. A follow-up gastrointestinal endoscopy performed two months later showed a polypoid lesion at the same place where the ulcer was previously (Fig. 1). The biopsied specimen was histologically diagnosed as well to moderately differentiated tubular adenocarcinoma. Preoperative laboratory examination again revealed moderate anemia (hemoglobin: $9.3 \mathrm{~g} / \mathrm{dL}$ ). He was positive for the serum anti-Helicobacter pylori $\mathrm{IgG}$ antibody $(17 \mathrm{U} / \mathrm{mL})$. Serum levels of tumor markers, including carcinoembryonic antigen (CEA), carbohydrate antigen (CA) 19-9, and CA 125, were in normal range. Computed tomography $(\mathrm{CT})$ of the abdomen revealed a protruding, $2.1 \times 1.5-\mathrm{cm}$ lesion inside the gastric body (Fig. 2). The patient underwent a laparoscopic distal gastrectomy with D2 lymph node dissection under the diagnosis of gastric adenocarcinoma.

A polypoid tumor measuring $28 \times 15 \mathrm{~mm}$ was found at the gastric body (Fig. 3). Microscopic examination of the resected specimens (hematoxylin-eosin stain) showed the tumor to include both carcinoma and sarcoma components (Fig. 4a). There was fibrosis indicating the ulcer scar in the submucosal layer. The carcinoma component predominated and was composed of well to moderately differentiated adenocarcinoma. The sarcoma component mainly consisted of atypical spindle cells 


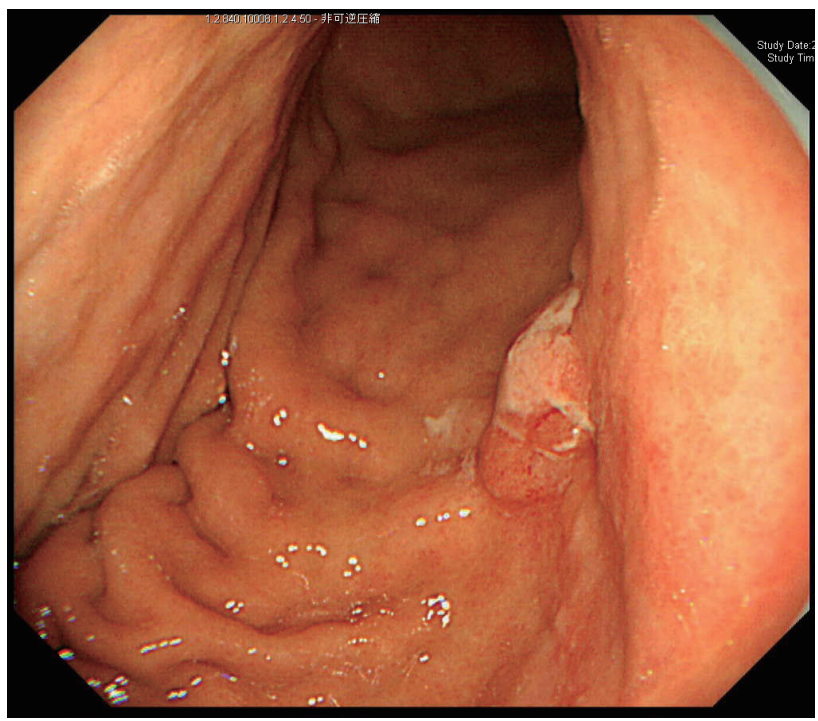

Fig. 1. A gastrointestinal endoscopy showing a polypoid lesion at the posterior wall of gastric body.

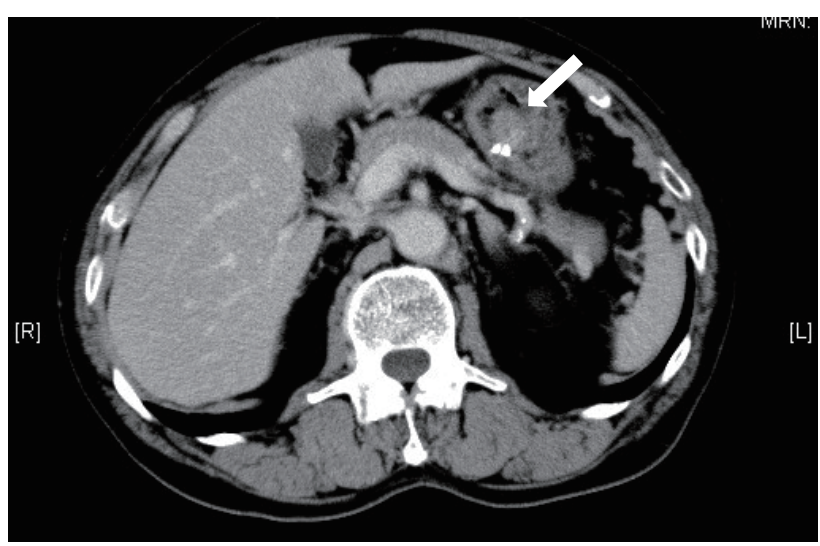

Fig. 2. A computed tomography of the abdomen revealed a protruded lesion (White arrow) measuring $2.1 \times 1.5 \mathrm{~cm}$ at the gastric body.

with frequent mitotic activity. It also contained a few carcinoma components. Transition between malignant epithelial and spindle cells was evident (Fig 4b). No chondrosarcoma, osteosarcoma or rhabdomyosarcoma components were observed anywhere. Immunohistochemical (IHC) staining revealed that spindle cells were positive for $\alpha$-smooth muscular actin (SMA; Fig. 5a), calponin (Fig. 5b), and h-caldesmon, but negative for cluster of differentiation (CD) 34, CD117 (c-kit), desmin, and dog 1. Furthermore, the carcinoma components were positive for cytokeratin AE1/3 (Fig. 6a), whereas the sarcoma components contained only a few cells positive for cytokeratin AE1/3 (Fig. 6b). These IHC findings led to a diagnosis of gastric carcinosarcoma (adenocarcinoma and leiomyosarcoma). The tumor was confined to

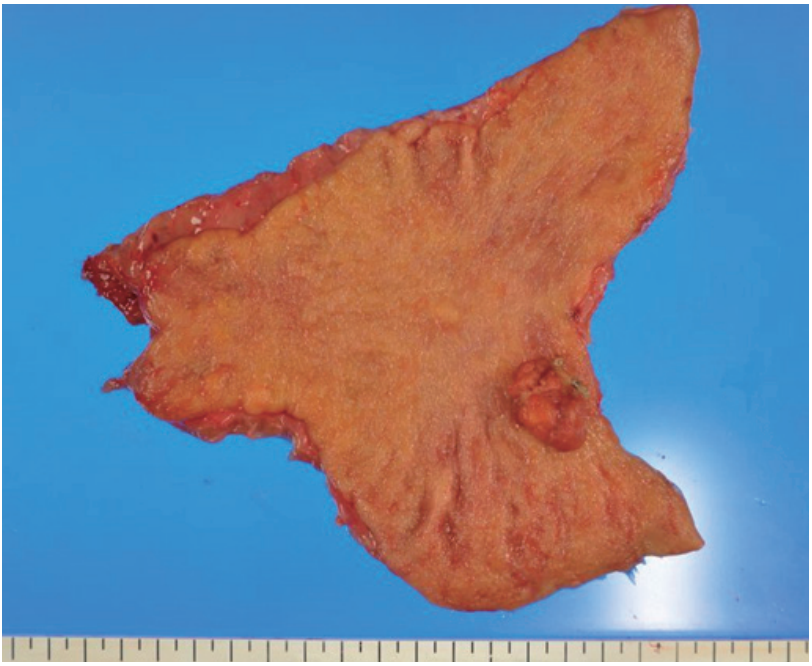

Fig. 3. Resected specimen. There was a polypoid tumor measuring $28 \times 15 \mathrm{~mm}$ at the gastric body.

the gastric mucous. Lymph node metastasis was found in one of the 70 dissected nodes and contained only the carcinoma component. According to the Japanese Classification of Gastric Cancer, ${ }^{11}$ the pTNM classification was T1a N1 M0 Stage 1b R0. His postoperative course was uneventful; he did not undergo postoperative adjuvant chemotherapy. He survived without recurrence for 2 years.

\section{DISCUSSION}

Queckenstedt described the first case of carcinosarcoma of the stomach in $1904 .^{12}$ Since then, 72 cases of gastric carcinosarcoma have been reported to date in the Japanese and English scientific literature. ${ }^{2-8}$ Table 1 shows the clinicopathologic characteristics of these 72 reported cases of gastric carcinosarcoma. Carcinosarcoma of the stomach is much more common in men $(n=50)$ than women $(n=22)$. The average age at diagnosis was 62.5 \pm 3.0 years old. Macroscopic appearance is often as a huge polypoid mass; the average tumor size is $8.6 \pm 1.0$ $\mathrm{cm}$.

As its clinical symptoms do not seem to differ from gastric adenocarcinomas, distinguishing carcinosarcoma of the stomach from gastric adenocarcinoma through endoscopy or radiology can be difficult. ${ }^{1}$ Histologically, the most common carcinomatous component of this tumor is tubular or papillary adenocarcinoma, whereas leiomyosarcoma, osteosarcoma, chondrosarcoma or rhabdomyosarcoma have been reported as sarcoma components. ${ }^{13-16}$

Although the pathogenesis of gastric carcinosarcoma remains controversial, two hypotheses have been 
(a)

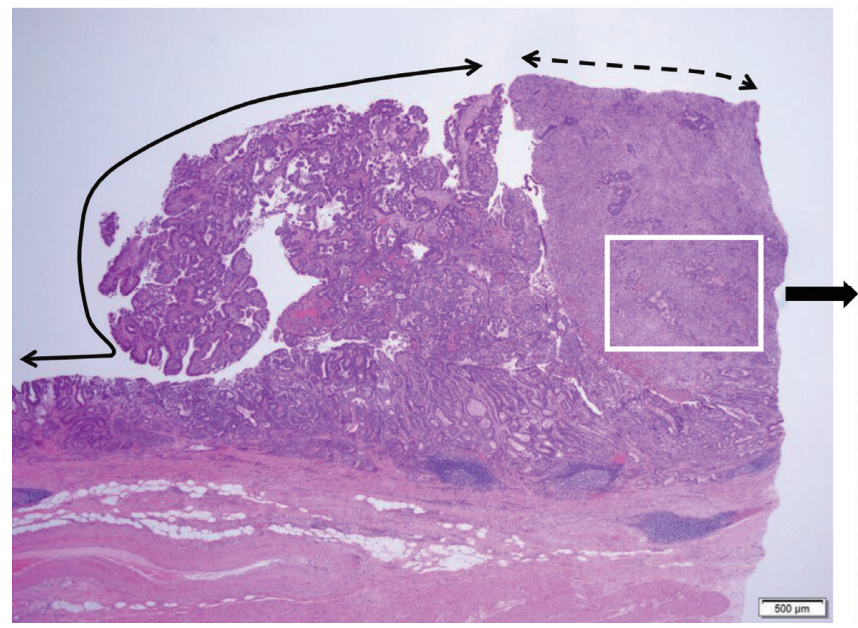

(b)

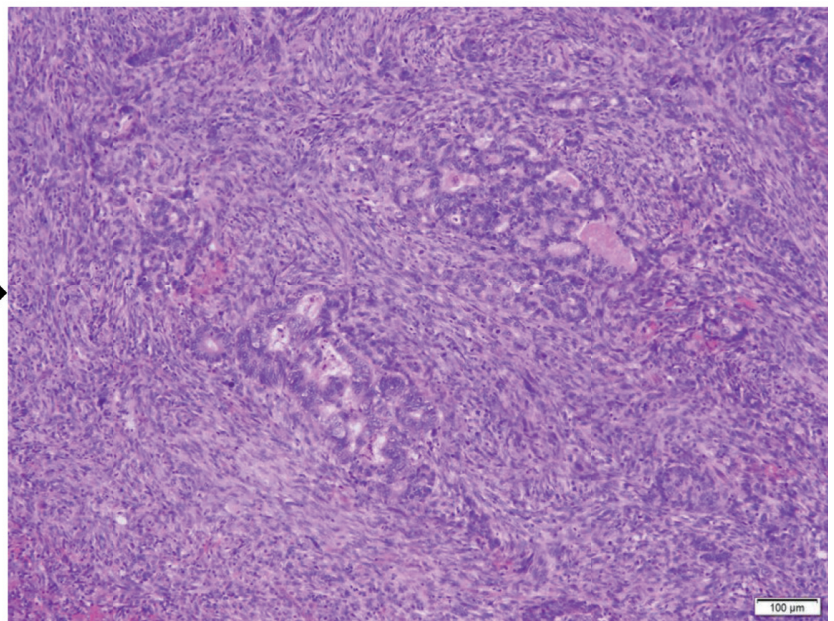

Fig. 4. Photomicrographs of hematoxylin-eosin-stained tissue sections of the tumor. (a) The tumor consisted of both carcinoma (solid line) and sarcoma components (dotted line) $($ Bar $=500 \mu \mathrm{m})$. (b) High-magnification image of the area surrounded by a white line. Transition between malignant epithelial and spindle cells was evident $(\mathrm{Bar}=100 \mu \mathrm{m})$.

(a)

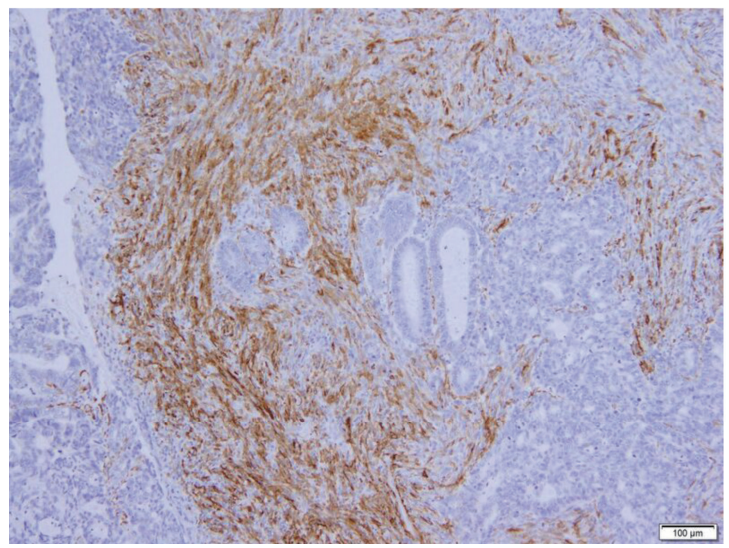

(b)

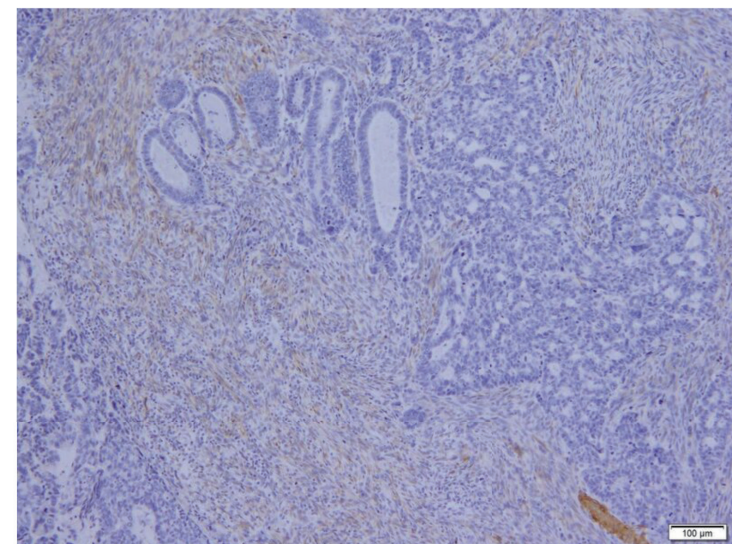

Fig. 5. Immunohistochemical staining revealed that tumor cells were positive for $\alpha$-smooth muscular actin $(\mathbf{a})$ and calponin $(\mathbf{b})($ Bar $=$ $100 \mu \mathrm{m})$.

(a)

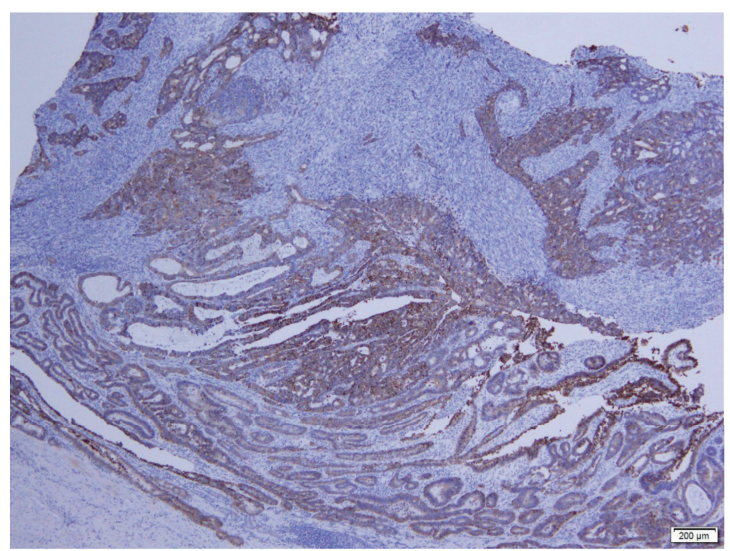

(b)

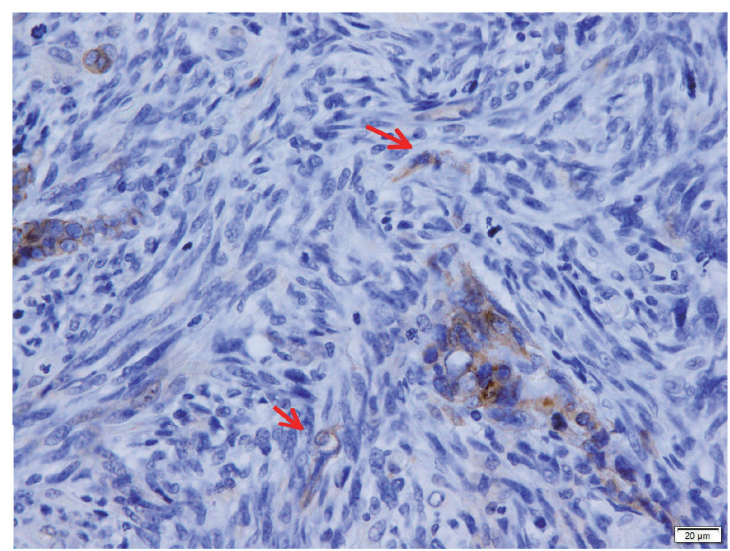

Fig. 6. Immunohistochemical staining of cytokeratin AE1/3. (a) The carcinoma components were positive for cytokeratin AE1/3 $(\mathrm{Bar}=$ $200 \mu \mathrm{m}$ ). (b) The sarcoma components contained only a few cells positive for cytokeratin AE1/3 (Red arrows; Bar $=20 \mu \mathrm{m}$ ). 
proposed. ${ }^{17}$ The first is the bi-clonal origin hypothesis, which supports the collision tumor theory, according to which the carcinosarcoma originates from two different tumor cell clones. The second is the monoclonal origin hypothesis, whereby the carcinosarcoma originates from a stem cell that can undergo both epithelial and mesenchymal differentiation. In our case, transition between malignant epithelial and spindle cells was evident, which supports the monoclonal origin hypothesis. IHC analysis was useful for a definitive diagnosis of carcinosarcoma. However, preoperative diagnosis of this tumor seems to be extremely difficult, possibly due to limited amount of biopsy specimen. In fact, the diagnosis of gastric carcinosarcoma tends to be established either postoperatively or at autopsy, whereas only 4 cases were reported in which diagnoses of gastric carcinosarcoma were made based on preoperative biopsy specimens..$^{14,18-21}$

Radical gastrectomy is regarded as the only curative treatment for gastric carcinosarcoma. However, this tumor tends to develop rapidly and to be diagnosed at an advanced clinical stage (Table 1). It therefore has a worse prognosis than other types of gastric carcinoma, even after radical gastrectomy. ${ }^{14}$ The mean survival period is estimated to be 7-10 months. ${ }^{3,17,22}$ The most frequently observed distant metastasis site was the liver (Table 1). Reportedly, more than $50 \%$ of tumor recurrences occur in the first postoperative year. ${ }^{17}$ Therefore, diagnosis and treatment as early as possible is crucial. To our knowledge, however, only four cases were diagnosed at early stage; in fact, our case was the first in which the tumor was confined to gastric mucosa. Table 2 shows the clinicopathologic characteristics of early carcinosarcoma of the stomach. ${ }^{21,23,24}$ Notably, lymph node metastasis was observed in two of four cases, which indicates the aggressiveness of this type of tumor. Therefore, chemotherapy and radiation therapy as well as surgery may play important roles in its treatment. However, as gastric carcinosarcoma is very rare, the effects of chemotherapy and radiotherapy have not yet been reported.

Carcinosarcoma of the stomach is a rare tumor with high malignant potential and a tendency towards poor prognosis. Thus, gastric carcinosarcoma should be considered as a part of differential diagnosis. IHC staining is critical in making an accurate diagnosis of carcinosarcoma. Careful follow up is required for early detection of recurrence, especially in the first postoperative year.

Ethics approval and consent to participate: Consent for publication was obtained from the patient.

The authors declare no conflict of interest.
Table 1. Summary of 72 previously reported cases of gastric carcinosarcoma

\begin{tabular}{|c|c|c|}
\hline Gender & $\begin{array}{l}\text { Male } \\
\text { Female }\end{array}$ & $\begin{array}{l}50 \\
22\end{array}$ \\
\hline Age (average \pm SD) & $62.5 \pm 3.0$ & \\
\hline \multirow[t]{8}{*}{ Symptom } & Anemia & 24 \\
\hline & Abdominal pain & 20 \\
\hline & Hematemensis or melena & 12 \\
\hline & Appetite loss & 9 \\
\hline & Dysphagia & 6 \\
\hline & None & 3 \\
\hline & Other symptoms & 14 \\
\hline & Not mentioned & 1 \\
\hline \multirow[t]{6}{*}{ Location } & Upper third & 21 \\
\hline & Middle third & 17 \\
\hline & Lower third & 24 \\
\hline & Whole & 5 \\
\hline & Gastric stump & 2 \\
\hline & Not mentioned & 3 \\
\hline Tumor size (average \pm SD) & $8.6 \pm 1.0 \mathrm{~cm}$ & \\
\hline \multirow{5}{*}{$\begin{array}{l}\text { Preoperative diagnosis ac- } \\
\text { cording to biopsy specimen }\end{array}$} & Adenocarcinoma & 24 \\
\hline & Carcinosarcoma & 4 \\
\hline & Sarcoma & 4 \\
\hline & Other & 6 \\
\hline & Not mentioned & 34 \\
\hline \multirow[t]{4}{*}{ Macroscopic appearance } & Protruded & 39 \\
\hline & Ulcerated & 24 \\
\hline & SMT like & 3 \\
\hline & Not mentioned & 6 \\
\hline \multirow[t]{7}{*}{ Depth of invasion $^{\text {a }}$} & M & 1 \\
\hline & $\mathrm{SM}$ & 3 \\
\hline & MP & 4 \\
\hline & SS & 19 \\
\hline & SE & 12 \\
\hline & SI & 8 \\
\hline & Not mentioned & 25 \\
\hline \multirow[t]{3}{*}{ Lymph node metastasis } & Absent & 17 \\
\hline & Present & 28 \\
\hline & Not mentioned & 27 \\
\hline \multirow[t]{9}{*}{ Distant metastasis } & Absent & 18 \\
\hline & Present & 29 \\
\hline & Liver metastasis & 19 \\
\hline & Lung metastasis & 3 \\
\hline & Distant Lymph node metastasis & 2 \\
\hline & Peritoneal metastasis & 2 \\
\hline & Other & 2 \\
\hline & Unknown & 4 \\
\hline & Not mentioned & 25 \\
\hline
\end{tabular}

apepth of invasion: M, tumor invasion of the lamina propria; SM, tumor invasion of the submucosa; MP, tumor invasion of the muscularis propria; SS, tumor invasion of the subserosa; SE, tumor penetration of the serosa; SI, tumor invasion of adjacent organs. 
Table 2. Summary of carcinosarcomas of the stomach confined to submucosa

\begin{tabular}{lllllllll}
\hline Author & Gender & Age & Location & \multicolumn{2}{l}{$\begin{array}{l}\text { Depth of inva- Macroscopic } \\
\text { sion }^{\mathrm{a}}\end{array}$} & $\begin{array}{l}\text { Tumor size } \\
\text { appearance }\end{array}$ & $\begin{array}{l}\text { Lymph node } \\
(\mathrm{cm})\end{array}$ & $\begin{array}{l}\text { Distant metas- } \\
\text { metastasis }\end{array}$ \\
\hline Fujii & M & 72 & Body & SM & Type 1 & $2.0 \times 1.8$ & Absent & Absent \\
Yuasa & F & 53 & Antrum & SM & Type 1 & $5.0 \times 4.5$ & Present & Absent \\
Uno & M & 65 & Pylorus & SM & Type 1 & $9.5 \times 7.0$ & Absent & Absent \\
Our case & M & 78 & Body & M & Type 0-I & $2.8 \times 1.5$ & Present & Absent \\
\hline
\end{tabular}

${ }^{a} \mathrm{M}$ : Tumor confined to the mucosa; SM: tumor confined to the submucosa

'Type 1: Polypoid tumors, sharply demarcated from the surrounding mucosa; Type 0-I: polypoid tumors

\section{REFERENCES}

1 Bansal M, Kaneko M, Gordon RE. Carcinosarcoma and separate carcinoid tumor of the stomach. A case report with light and electron microscopic studies. Cancer. 1982;50:1876-81. PMID: 7116311.

2 Fujiie M, Yamamoto M, Taguchi K, Iwanaga A, Ohgaki K, Egashira A, et al. Gastric carcinosarcoma with rhabdomyosarcomatous differentiation: a case report and review. Surg Case Rep. 2016;2:52. PMID: 27250580.

3 Maeda O, Ando T, Ishiguro K, Watanabe O, Miyahara R, Miyata T, et al. A case of gastric carcinosarcoma with distant metastasis for which chemotherapy with S-1 plus cisplatin was transiently effective. International Cancer Conference Journal. 2014;3:252-9.

4 Kuwahara M, Kitamura K, Muramoto T, Kaneko K, Nozu F, Yoshikawa N, et al. A case of "so-called carcinosarcoma" of the stomach with anemia. Progress of Digestive Endoscopy. 2004;65:76-7.

5 Pinto JA, Gonzalez-Alfonso JE, Gonzalez L, Gonzalez L, Stevenson N. Well differentiated gastric adenocarcinoma with rhabdoid areas: a case report with immunohistochemical analysis. Pathol Res Pract. 1997;193.

6 Liu SW, Chen GH, Hsieh PP. Collision tumor of the stomach: a case report of mixed gastrointestinal stromal tumor and adenocarcinoma. J Clin Gastroenterol. 2002;35:332-4. PMID: 12352297.

7 Jang SM, Jang S-H, Min K-W, Na W, Jun YJ, Paik SS. A case of gastric carcinosarcoma with neuroendocrine and smooth muscle differentiation. Korean J Pathol. 2010;44:87-91.

8 Gohongi T, Iida H, Gunji N, Orii K, Ogata T. Postsurgical radiation therapy for gastric carcinosarcoma with c-kit expression: a case report. World J Gastroenterol. 2015;21:2830-5. PMID: 25759557.

9 Kawabata Y, Nakai T, Ooba K, Okumura T, Matsuura Y, Miyazaki Y. A case report of carcinosarcoma of the stomach. Jpn J Gastroenterol Surg. 1993;26:2189-93.

10 Yamazaki K. A gastric carcinosarcoma with neuroendocrine cell differentiation and undifferentiated spindle-shaped sarcoma component possibly progressing from the conventional tubular adenocarcinoma; an immunohistochemical and ultrastructural study. Virchows Arch. 2003;442:77-81. PMID: 12536318.

11 Japanese classification of gastric carcinoma: 3rd English edition. Gastric Cancer. 2011;14:101-12. PMID: 21573743.

12 Saphir, Vass A. Carcinosarcoma. Am J Cancer. 1938;33:33161.

13 Matsukuma S, Wada R, Hase K, Sakai Y, Ogata S, Kuwabara N. Gastric stump carcinosarcoma with rhabdomyosarcoma- tous differentiation. Pathol Int. 1997;47:73-7. PMID: 9051696.

14 Nakayama Y, Murayama H, Iwasaki H, Iwanaga S, Kikuchi $\mathrm{M}$, Ikeda S, et al. Gastric carcinosarcoma (sarcomatoid carcinoma) with rhabdomyoblastic and osteoblastic differentiation. Pathol Int. 1997;47:557-63. PMID: 9293537.

15 Tsuneyama K, Sasaki M, Sabit A, Yokoi K, Arano Y, Imai $\mathrm{T}$, et al. A case report of gastric carcinosarcoma with rhabdomyosarcomatous and neuroendocrinal differentiation. Pathol Res Pract. 1999;195:93-7; discussion 8. PMID: 10093828.

16 Teramachi K, Kanomata N, Hasebe T, Ishii G, Sugito M, Ochiai A. Carcinosarcoma (pure endocrine cell carcinoma with sarcoma components) of the stomach. Pathol Int. 2003;53:552-6. PMID: 12895235.

17 Cirocchi R, Trastulli S, Desiderio J, Grassi V, Barillaro I, Santoro A, et al. Gastric carcinosarcoma: A case report and review of the literature. Oncol Lett. 2012;4:53-7. PMID: 22807959

18 Takase T, Harada A, Yaguchi T, Kajikawa M, Nakayama S, Shirota T, et al. A Case of True Gastric Carcinosarcoma Diagnosed Preoperatively by Endoscopic Biopsies. The Japanese Journal of Gastroenterological Surgery. 2006;39:294-9.

19 Ishiba T, Inokuchi M, Enjoji M, Ohno R, Ogawa N, Sugita $\mathrm{H}$, et al. A Case of AFP-Producing, True Gastric Carcinosarcoma. The Japanese Journal of Gastroenterological Surgery. 2013;46:814-21.

20 Randjelovic T, Filipovic B, Babic D, Cemerikic V, Filipovic B. Carcinosarcoma of the stomach: a case report and review of the literature. World J Gastroenterol. 2007;13:5533-6. PMID: 17907304.

21 Uno G, Miyaoka Y, Hukazawa K, Kamiyama S, Hujishiro $\mathrm{H}$, Kouge N, et al. A case report of gastric carcinosarcoma with abdominal fullness caused by invagination of the tumor into duodenal bulbus. Gastroenterological Endoscopy. 2011;53:3272-9.

22 Sato Y, Shimozono T, Kawano S, Toyoda K, Onoe K, Asada Y, et al. Gastric carcinosarcoma, coexistence of adenosquamous carcinoma and rhabdomyosarcoma: a case report. Histopathology. 2001;39:543-4. PMID: 11737318.

23 Yuasa Y, Okitsu H, Furukawa T, Kihara A, Yamashita M, Okitsu N. A case of true gastric carcinosarcoma treated using laparoscopy-assisted distal gastrectomy. Nihon Rinsho Geka Gakkai Zasshi (Journal of Japan Surgical Association). 2012;73:1110-4.

24 Fujii H, Iwase K, Higaki J, Mikata S, Imakita M, Kamiike W. A case of gastric carcinosarcoma accompanied by gastric gastrointestinal stromal tumor. Nihon Rinsho Geka Gakkai Zasshi (Journal of Japan Surgical Association). 2002;63:29347. 